\title{
OBSERVATIONAL COSMOLOGY
}

\section{ADELAIDE HEWITT, GEOFFREY BURBIDGE, and LI ZHI FANG (EDS.)}

\begin{abstract}
Observational Cosmology - the study of the radiation and matter which make up the universe and how they evolve with time, has become an increasingly active field of astronomical investigation.

In this volume up-to-date results on the important quantities which can be measured are described and critically discussed. They include the cosmic microwave background and its degree of isotropy, the rate of expansion of the universe, the Hubble constant, and the deceleration parameter $q_{0}$, the large-scale distribution of galaxies, the evidence for dark matter, and the distribution and properties of quasi-stellar objects. Radio, infrared, optical, and X-ray observations are all reported. There is also a full discussion of gravitational lenses, and a report of observations which do not fit into conventional theories.
\end{abstract}

